\title{
MYC promotes the development of papillary thyroid carcinoma by inhibiting the expression of IncRNA PAX8-AS1:28
}

\author{
YI ZHANG ${ }^{1}$, FEILEI LI ${ }^{1}$ and JUN CHEN ${ }^{2}$ \\ ${ }^{1}$ Department of Neck Surgery, The Second Affiliated Hospital of Wenzhou Medical University; \\ ${ }^{2}$ Department of Immunology, Wenzhou Medical University, Wenzhou, Zhejiang 325000, P.R. China
}

Received January 15, 2018; Accepted December 13, 2018

DOI: 10.3892/or.2019.6996

\begin{abstract}
As a common malignancy of the endocrine system, papillary thyroid carcinoma (PTC) seriously affects the quality of life of patients. IncRNA PAX8-AS1:28, or lnc-PSD4-1:14 has been reported to be abnormally expressed in PTC. However, the function of PAX8-AS1:28 in PTC is still unknown. Therefore, the present study aimed to investigate the functions of PAX8-AS1:28 in PTC, and to explore the possible mechanisms of action. A total of 38 patients with PTC were included and the normal thyroid follicular epithelial cell line Nthy-ori 3-1 and PTC cell line IHH-4 were also used. MYC and PAX8-AS1:28 overexpression and siRNA silencing in the cell lines were carried out. Expression of PAX8-AS1:28, PAX8 and MYC in tumor tissue, adjacent healthy tissue and different cell lines were detected by qRT-PCR and western blot analysis. Cell proliferation was measured by CCK- 8 assay. Expression levels of PAX8-AS1:28 and PAX8 were lower in PTC tumor tissue and PTC cells than those in healthy tissue and normal cells. In contrast, the expression level of MYC was higher in PTC cells than that in normal cells. PAX8-AS1:28 silencing reduced the expression level of PAX8 and promoted tumor cell growth, while PAX8-AS1:28 overexpression increased the expression level of PAX8 and inhibited tumor cell growth. MYC silencing increased expression levels of PAX8-AS1:28 and PAX8 and inhibited tumor cell growth, while MYC overexpression decreased expression levels of PAX8-AS1:28 and PAX8 and promoted tumor cell growth. MYC can promote PTC by inhibiting the expression of 1ncRNA PAX8-AS1:28.
\end{abstract}

\section{Introduction}

Apart from medullary carcinoma, all other types of thyroid cancers originate from follicular cells, which form the simple

Correspondence to: Dr Jun Chen, Department of Immunology, Wenzhou Medical University, Chashan Higher Education Park, Ouhai, Wenzhou, Zhejiang 325000, P.R. China

E-mail:prpecjzz@yeah.net

Key words: papillary thyroid carcinoma, lncRNA, PAX8-AS1:28, siRNA, expression, inhibition unicellular epithelium of the thyroid (1). In spite of the development of preventive measures, the incidence of thyroid cancer has increased more than 3 -fold during the past 3 decades, and this disease tends to affect younger patients (2). As the most common type of thyroid cancer, papillary thyroid carcinoma (PTC) accounts for more than $80 \%$ of the total cases of this disease (3). With the development of treatment strategies such as radiotherapy, chemotherapy, thyroid hormone treatment, surgical resection and combined treatment, the 5-year survival rate of patients with PTC has now reached 95\% (4). However, due to the unclear pathogenesis, the recurrence rate of this disease is still high (5). More importantly, PTC may occasionally dedifferentiate into more aggressive and lethal thyroid cancers (1). Therefore, the in-depth understanding of the pathogenesis of this disease is highly needed.

Long non-coding RNA, which is also known as lncRNA, is a class of non-coding RNAs with a length longer than 200 nucleotides, which is significantly longer than that of short interfering RNAs, microRNAs and other short RNAs (6). Numerous studies have shown that different lncRNAs have their specific functions in normal biological or pathological processes (7). In addition to this, quite a few of lncRNAs were proven to play essential roles in the development and progression of a variety of human diseases including liver diseases, heart diseases, and different types of human cancer (8-10). A recent study showed that lncRNA PAX8-AS1:28, or lnc-PSD4-1:14, is abnormally expressed in PTC (11), indicating the involvement of lncRNA PAX8-AS1:28 in this disease. However, the role of lnc-PSD4-1:14 in PTC is still unknown.

In the present study, expression of lncRNA PAX8-AS1:28 in PTC tissues and adjacent healthy tissues were detected. The relationships between PAX8-AS1:28, PAX8 and MYC were investigated.

\section{Materials and methods}

Patients. A total of 38 patients with PTC were enrolled in our hospital from October 2016 to October 2017. These patients included 13 males and 25 females, and the ages ranged from 20 to 78 years with an average age of 40.2 years. All patients were diagnosed by the standards that have been established by the World Health Organization. Patients with thyroid microcarcinoma were excluded. All patients received surgical resections, and tumor tissues as well as adjacent healthy tissues 
were collected during the surgical operation. All patients had normal thyroid function before surgery, and none of them received radiotherapy or chemotherapy before admission. The Ethics Committee of the Second Affiliated Hospital of Wenzhou Medical University approved this study. All patients provided informed consent.

Cell lines and cell culture. Normal thyroid follicular epithelial cell line Nthy-ori 3-1 and PTC cell line IHH-4 were purchased from the American Type Culture Collection (ATCC, Manassas, VA, USA). Cells were cultured with DMEM containing $10 \%$ FBS, $100 \mathrm{mg} / \mathrm{ml}$ penicillin $\mathrm{G}$ and $100 \mathrm{U} / \mathrm{ml}$ streptomycin (all from Invitrogen; Thermo Fisher Scientific, Inc., Waltham, MA, USA) in an incubator at $37^{\circ} \mathrm{C}$ in $5 \% \mathrm{CO}_{2}$.

Real-time quantitative PCR. Trizolreagent(Invitrogen; Thermo Fisher Scientific) was used for total RNA extraction. The quality of RNA samples was tested using NanoDrop ${ }^{\mathrm{TM}} 2000$ spectrophotometers (Thermo Fisher Scientific), and only those with an A260/A280 ratio between 1.8 and 2.0 were used in reverse transcription to synthesize cDNA by using Oligo(dT) $)_{15}$ (Sangon, Shanghai, China) and AMV reverse transcriptase (Gibco; Thermo Fisher Scientific). TaqMan PCR kit (Thermo Fisher Scientific) was used for PCR reaction. The PCR reaction was performed on Bio-Rad iCycler (Bio-Rad Laboratories, Inc., Hercules, CA, USA). The following primers were used in the PCR reactions: 5'-GGCTTTGTGCTACTGCTTCA-3' (forward) and 5'-TCTAACCCTCCTGGCTTCCT-3' (reverse) for PAX8-AS1:28; 5'-GATCAGGATAGCTGCCGACT-3' (forward) and 5'-GTTGTACCTGCTCGCCTTTG-3' (reverse) for PAX8; 5'-CAGGAGGCATTGCTGATGAT-3' (forward) and 5'-GAAGGCTGGGGCTCATTT-3' (reverse) for GAPDH; 5'-GCCACGTCTCCACACATCAG-3' (forward) and 5'-TCT TGGCAGCAGGATAGTCCTT-3' (reverse) for MYC. PCR reaction conditions were: $95^{\circ} \mathrm{C}$ for $1 \mathrm{~min}$, followed by 40 cycles of $95^{\circ} \mathrm{C}$ for $15 \mathrm{sec}$ and $56^{\circ} \mathrm{C}$ for $35 \mathrm{sec} . \mathrm{Ct}$ values were processed using the $2^{-\Delta \Delta \mathrm{C}_{\mathrm{T}}}$ method, and relative expression level of each gene was normalized to endogenous control GAPDH.

Construction of IncRNA PAX8-AS1:28 and MYC expression vector and transfection. Cloning primers for PAX8-AS1:28 were 5'-CGTGTCGACCTCATTCATTTGTTC-3' (forward) and 5'-CGAAAGCTTAAAAAACTGACACATGC-3' (reverse). Cloning primers for MYC were 5'-CGAAAGCT TGCCACCATGCTCGGAAGGACTATCCTGCTGCCAA-3' (forward) and 5'-CGTGGATCCGGCGCTCCAAGACGT TGTGTGTTCG-3' (reverse). PCR amplified fragments with the U6 promoter were inserted into the pcDNA3.1 vector. IHH- 4 cells were collected during the logarithmic growth phase, and were seeded in 6 -well plates with $4.5 \times 10^{5}$ cells per well. Cells were cultured in an incubator $\left(37^{\circ} \mathrm{C}, 5 \% \mathrm{CO}_{2}\right)$. Five micrograms of PAX8-AS1:28 and MYC expression plasmid was diluted in $250 \mu \mathrm{l}$ of serum-free medium and transfection was performed using Invitrogen ${ }^{\mathrm{TM}}$ Lipofectamine 2000 transfection reagent (cat. no. 11668-019, Thermo Fisher Scientific). Medium was replaced 4-6 h after transfection. After incubation for another $48 \mathrm{~h}$, the cells were collected for subsequent experiments. Expression of PAX8-AS1:28 and MYC was detected after transfection to confirm the success of transfection.
siRNA transfection. MYC siRNA (cat. no. AM16708) and control siRNA (cat. no. AM4611) were purchased from Applied Biosystems/ThermoFisherScientific.ThePAX8-AS1:28siRNA target sequence was GAGAGGTCATTATGTGAAGGCT. siRNA (final concentration, $50 \mathrm{nM}$ ) was transfected into IHH-4 cells using Lipofectamine 2000 transfection reagent (cat. no. 11668-019, Thermo Fisher Scientific) according to the manufacturer's instructions. Medium was replaced 4-6 $\mathrm{h}$ after transfection. After incubation for another $48 \mathrm{~h}$, the cells were collected for subsequent experiments.

Cell proliferation assay. Cell counting kit (CCK-8; Sigma-Aldrich; Merck KGaA, Darmstadt, Germany) was used to measure cell proliferation ability according to the manufacturer's instructions. Briefly, $100 \mu \mathrm{l}$ of cell suspension containing $5 \times 10^{3}$ cells was transferred to each well of 96-well plates. Cells were cultured and CCK-8 solution (10 $\mu \mathrm{l})$ was added into each well 24, 48 and $72 \mathrm{~h}$ later. After incubation at $37^{\circ} \mathrm{C}$ for another $4 \mathrm{~h}$, optical density (OD) values at $450 \mathrm{~nm}$ were measured using a microplate reader (Bio-Rad, USA).

Western blot assay. Total protein was extracted using cell lysis buffer (P0013K, Beyotime Institute of Biotechnology, Haimen, China), and the concentration was determined by BCA assay. Then, $20 \mu \mathrm{g}$ of protein from each sample was subjected to $10 \%$ SDS-PAGE gel electrophoresis, followed by transmembrane to PVDF. Membranes were then blocked with $5 \%$ skimmed milk at room temperature for $1 \mathrm{~h}$. After washing with TBST, membranes were then incubated with primary antibodies including rabbit anti-PAX8 (dilution 1:2,000, cat. no. ab53490, Abcam), rabbit anti-c-Myc (dilution 1:2,000, cat. no. ab32072, Abcam) and rabbit anti- $\beta$-actin (dilution 1:2,000, cat. no. ab8227, Abcam) overnight at $4^{\circ} \mathrm{C}$. After washing with PBS, the membranes were further incubated with anti-rabbit IgG-HRP secondary antibody (dilution 1:1,000, cat. no. MBS435036, MyBioSource) at room temperature for $2 \mathrm{~h}$. Then the membranes were washed again with TBST, signals were detected using ECL (Sigma-Aldrich; Merck KGaA) method. Relative expression level of each protein was normalized to endogenous control $\beta$-actin using ImageJ software (National Institutes of Health, Bethesda, MD, USA).

Statistical analysis. Statistical analyses were performed using SPSS 19.0(IBM Corp.,Armonk, NY,USA). Normal distribution data were recorded as mean $\pm \mathrm{SD}$ ), and comparisons between two groups were performed by t-test. Correlation between the expression levels of AX8-AS1:28 and PAX8 mRNAs was analyzed by Spearman's rank correlation coefficient. $\mathrm{P}<0.05$ was considered to be statistically significant.

\section{Results}

Expression of PAX8-AS1:28 and PAX8 mRNAs in PTC tissues and adjacent normal tissues. qRT-PCR was performed to detect the expression of PAX8-AS1:28 and PAX8 mRNAs in PTC tissues and adjacent normal tissues. Compared with adjacent normal tissues, expression levels of PAX8-AS1:28 (Fig. 1A) and PAX8 (Fig. 1B) mRNAs were significantly reduced in PTC tissues $(\mathrm{P}<0.01)$. In addition, the expression level of PAX8-AS1:28 was positively correlated with expression of PAX8 (Fig. 1C, 

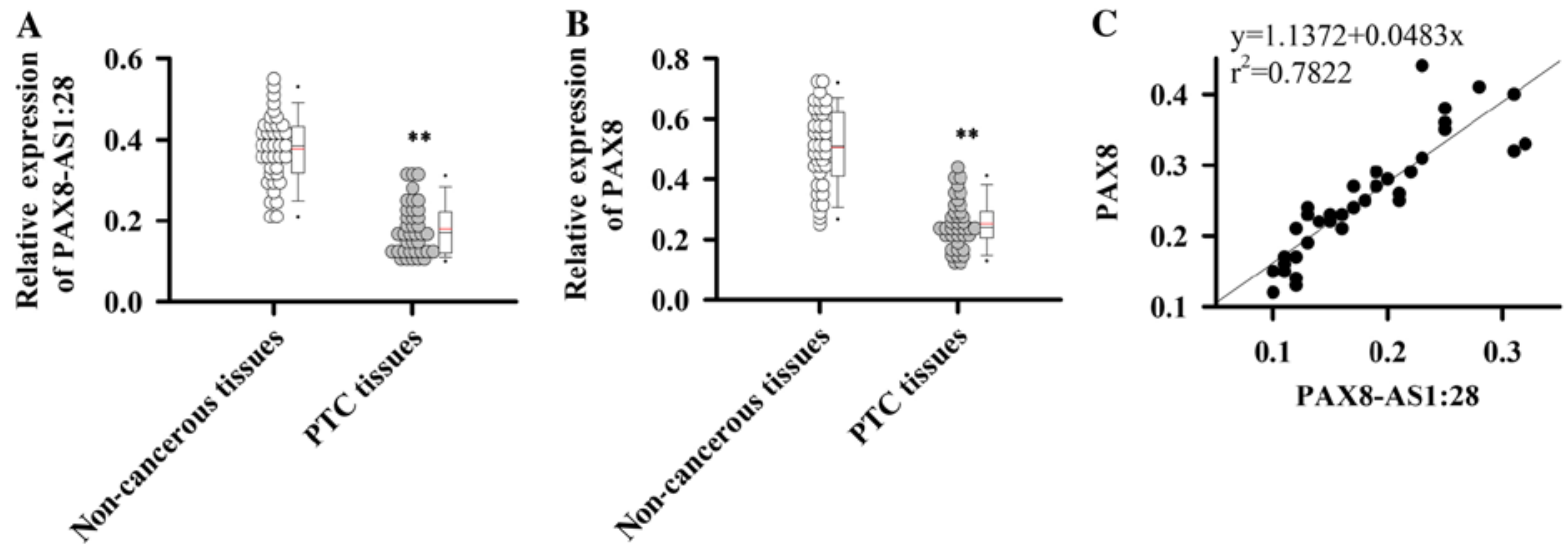

Figure 1. Expression of PAX8-AS1:28 and PAX8 mRNA in PTC and adjacent normal tissues. (A) Expression of PAX8-AS1:28 mRNA in PTC and adjacent normal tissues. GAPDH was used as an endogenous control. (B) Expression of PAX8 mRNA in PTC and adjacent normal tissues. GAPDH was used as an endogenous control. (C) Correlation between the expression of PAX8-AS1:28 and PAX8 mRNA. ${ }^{* *}$ P $<0.01$, compared with the control group.
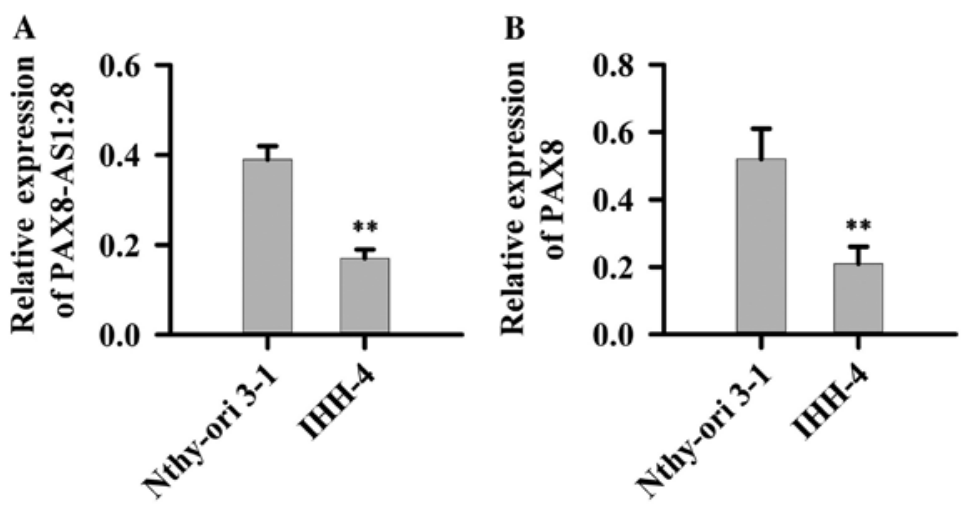

Figure 2. Expression of PAX8-AS1:28 and PAX8 in normal cell line and PTC cell line. (A) Expression of PAX8-AS1:28 in normal cell line Nthy-ori 3-1 and PTC cell line, IHH-4. GAPDH was used as an endogenous control. (B) Expression of PAX8 in normal cell line and PTC cell line. GAPDH was used as an endogenous control. ${ }^{* *} \mathrm{P}<0.01$, compared with the control group.

$\left.\mathrm{r}^{2}=0.7822\right)$. These data suggest that PAX8-AS1:28 and PAX8 are downregulated in PTC.

Expression of PAX8-AS1:28 and PAX8 in normal and PTC cells. Expression of PAX8-AS1:28 and PAX8 in normal cell line Nthy-ori 3-1 as well as PTC cell line IHH-4 was detected. Compared with normal cell line Nthy-ori 3-1, expression levels of PAX8-AS1:28 (Fig. 2A) and PAX8 mRNAs (Fig. 2B) were significantly reduced in the IHH-4 cells $(\mathrm{P}<0.01)$.

Effect of PAX8-AS1:28 overexpression and silencing on PAX8 expression and IHH-4 cell growth. After siRNA silencing, the expression level of PAX8-AS1 was significantly reduced (Fig. 3A), indicating the success of transfection. Compared with control cells, expression levels of PAX8 mRNA (Fig. 3A) and protein (Fig. 3B) were significantly reduced in IHH- 4 cells following PAX8-AS1:28 siRNA silencing. In addition, PAX8-AS1:28 siRNA silencing significantly promoted the growth of IHH-4 cells (Fig. 3C).

Cells transfected with the PAX8-AS1:28 plasmid show an elevated expression level of PAX8-AS1:28 (Fig. 3D), indicating the successfully established PAX8-AS1 overexpression cell line. Compared with the control cells, expression levels of PAX8 mRNA (Fig. 3D) and protein (Fig. 3E) were significantly increased in IHH-4 cells with PAX8-AS1:28 overexpression. In addition, PAX8-AS1:28 overexpression significantly inhibited the growth of IHH-4 cells (Fig. 3F). These data suggest that PAX8-AS1:28 can positively regulate the expression of PAX8 to inhibit PTC.

Expression of MYC in normal cell lines and PTC cell lines. Expression of MYC in normal cell line Nthy-ori 3-1 and PTC cell line IHH-4 was detected. Compared with normal cell line Nthy-ori 3-1, expression levels of MYC were significantly increased in thr IHH-4 cells $(\mathrm{P}<0.01$, Fig. 4).

Effects of MYC overexpression and silencing on PAX8 and PAX8-AS1:28 expression and IHH-4 cell growth. Cells transfected with the MYC plasmid showed elevated expression level of MYC (Fig. 5A and B), indicating the successfully established MYC overexpression cell line. Compared with the control cells, expression levels of PAX8-AS1:28 (Fig. 5A) and PAX8 (Fig. 5A and C) were significantly decreased in the IHH-4 cells with MYC overexpression. In addition MYC overexpression significantly promoted the growth of IHH-4 cells (Fig. 5D).

After siRNA silencing, expression level of MYC was significantly reduced (Fig. 5E and F), indicating the success of 
A
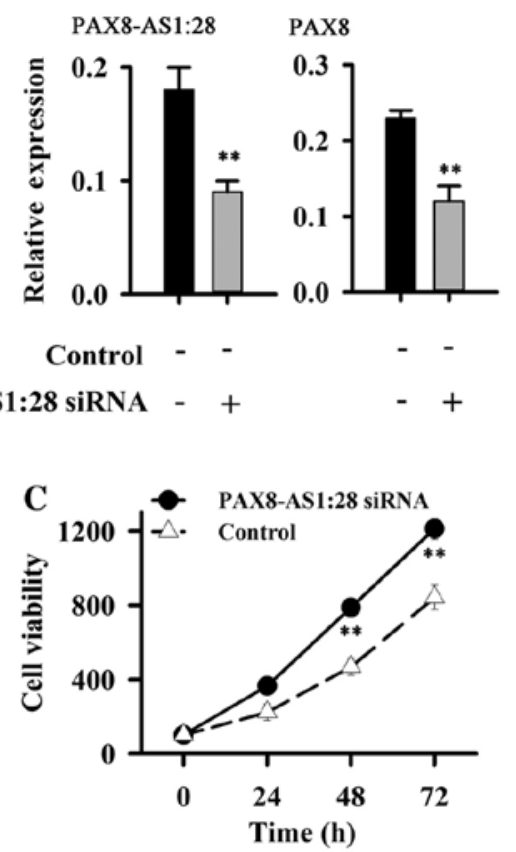

B

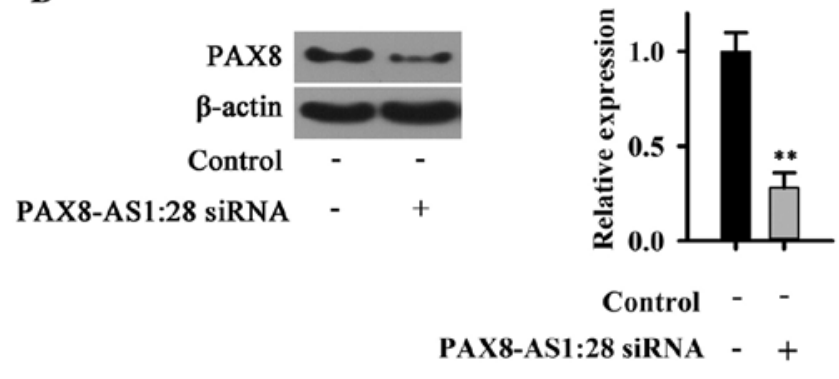

D

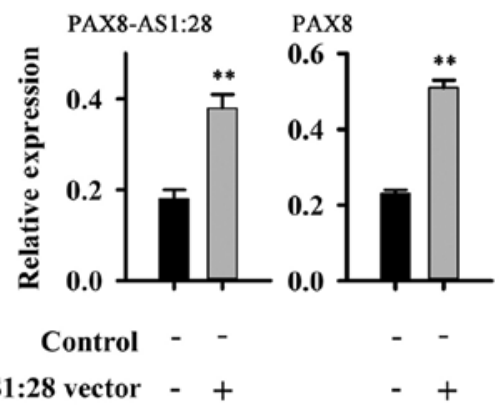

$\mathbf{E}$
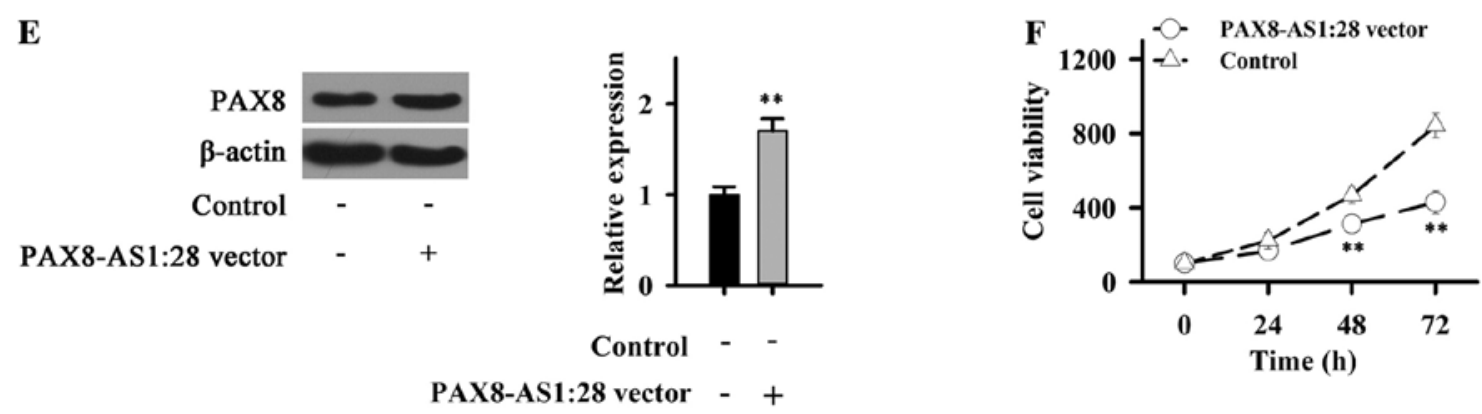

Figure 3. Effect of PAX8-AS1:28 overexpression and silencing on PAX8 expression and IHH-4 cell growth. (A) Effect of PAX8-AS1:28 siRNA silencing on PAX8-AS1:28 and PAX8 RNA expression. (B) Effect of PAX8-AS1:28 siRNA silencing on PAX8 protein expression. $\beta$-actin was used as an endogenous control. (C) Effect of PAX8-AS1:28 siRNA silencing on cell growth. (D) Effect of PAX8-AS1:28 overexpression on PAX8-AS1:28 and PAX8 RNA expression; (E) Effect of PAX8-AS1:28 overexpression on PAX8 protein expression. (F) Effect of PAX8-AS1:28 overexpression on cell growth. $\beta$-actin was used as a endogenous control. ${ }^{* *} \mathrm{P}<0.01$, compared with the control group.

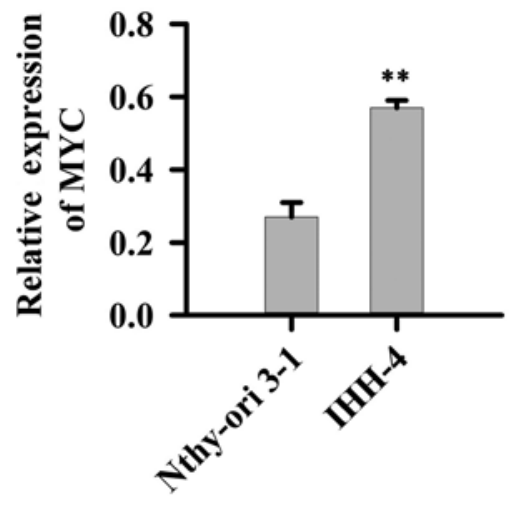

Figure 4. Expression of MYC in normal cell line Nthy-ori 3-1 and PTC cell line IHH-4. GAPDH was used as an endogenous control. ${ }^{* *} \mathrm{P}<0.01$, compared with the control group.

transfection. Compared with the control cells, expression levels of PAX8-AS1:28 (Fig. 5E) and PAX8 protein (Fig. 5G) were significantly reduced in IHH-4 cells following MYC siRNA silencing. In addition, MYC siRNA silencing significantly inhibited the growth of IHH-4 cells (Fig. 5H). These data suggest that MYC can negatively regulate the expression of PAX8-AS1:28 and PAX8 to promote PTC.

\section{Discussion}

The pathogenesis of PTC is still unclear. Therefore, understanding the molecular pathogenesis and mechanisms underlying PTC is still a 'hot research spot' regarding the treatment of PTC (12). Recent studies have identified multiple pathways that are related to the development and progression of PTC, such as the HIF-1 $\alpha$ pathway (13), the thyroid-stimulating hormone receptor signaling pathway (14) and the WNT/ $\beta$-catenin signaling pathway (15). Epigenetic and genetic alterations in those pathways, including altered gene copy-number, gene mutation and aberrant gene methylation play central roles in the pathogenesis of PTC (12). A variety of lnRNAs have also been proven to be involved in the development of PTC. IncRNA SLC6A9 was found to be downregulated in 131I-resistant PTC accompanied by the inhibition of PARP-1, and a high expression level of SLC6A9 

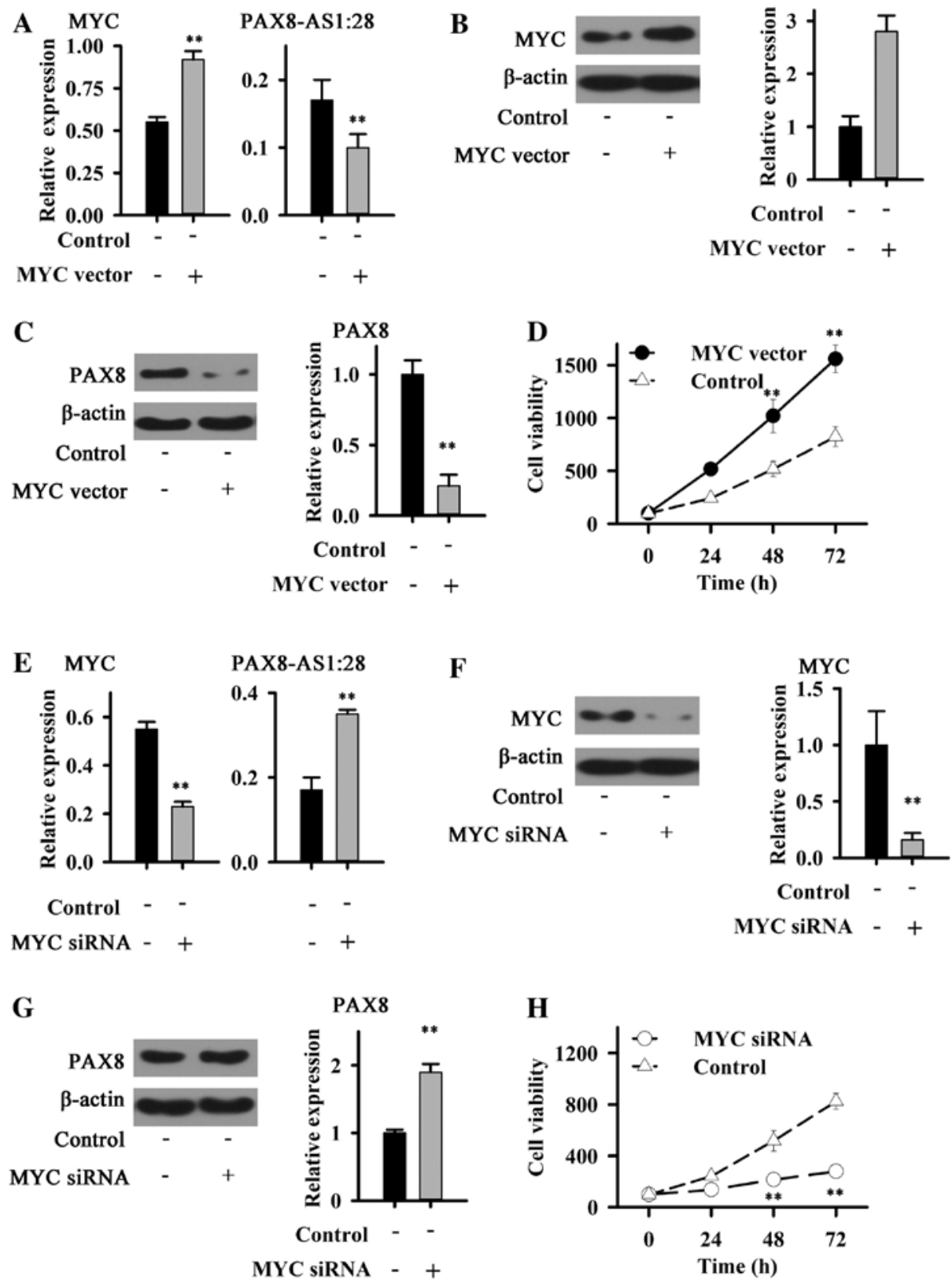

Figure 5. Effects of MYC overexpression and silencing on PAX8 and PAX8-AS1:28 expression and IHH-4 cell growth. (A) Effect of MYC overexpression on MYC mRNA and PAX8-AS1:28 RNA expression. (B) Effect of MYC overexpression on MYC protein expression. $\beta$-actin was used as an endogenous control. (C) Effect of MYC overexpression on PAX8 protein expression. $\beta$-actin was used as an endogenous control. (D) Effect of MYC overexpression on cell growth. (E) Effect of MYC silencing on MYC mRNA and PAX8-AS1:28 RNA expression. $\beta$-actin was used as an endogenous control. (F) Effect of MYC silencing on MYC protein expression. (G) Effect of MYC silencing on PAX8 protein expression. $\beta$-actin was used as an endogenous control. (H) Effect of MYC silencing on cell growth. ${ }^{* *} \mathrm{P}<0.01$, compared with the control group.

was found to be positively correlated with the overall survival rate and disease-free survival rate of PTC patients who received 131I therapy, indicating that SLC6A9 can potentially serve as a novel target for the treatment of 131I-resistant PTC (16). In addition to the direct effects on PTC, lncRNAs can also interact with key signal transduction pathways that are involved in the pathogenesis of PTC. In a recent study, lncRNA PTCSC was proven to significantly regulate the expression of SCAI and subsequently alter the activity of Wnt/ $\beta$-catenin signal transduction, which in turn regulated the proliferation and migration of PTC cells (17). All of these previous studies suggest that lncRNA are key players in the pathogenesis of PTC.
lnc-PSD4-1:14, or 1ncRNA PAX8-AS1:28, is a newly discovered lncRNA. Based on our knowledge, the functionality of lncRNA PAX8-AS1:28 is still unknown. It has been reported that the expression level of lncRNA PAX8-AS1:28 is commonly decreased in patients with neck squamous cell carcinoma, and a higher expression level of lncRNA PAX8-AS1:28 is closely correlated with better survival outcome (18). IncRNA PAX8-AS1:28 expression is also downregulated in patients with PTC (11), and the reduced expression level of lncRNA PAX8-AS1:28 is closely correlated with the poor survival of these patients (19). Consistent with previous studies, in our study, the expression level of PAX8-AS1:28 was found to be lower in PTC tissues and PTC cells than that in adjacent 
healthy tissues and a normal cell line. Our finding further confirmed the involvement of PAX8-AS1:28 in PTC.

Paired box gene 8, or PAX8, is a transcription factor that belongs to the paired box (PAX) family (20). Mutations in PAX8 have been proven to be related with various thyroid diseases including thyroid follicular carcinomas, thyroid dysgenesis and atypical follicular thyroid adenomas (20). lncRNA PAX8-AS1:28 overlaps with paired box 8 (PAX8) in an antisense orientation (18), indicating the possible interactions between PAX8 and 1ncRNA PAX8-AS1:28. In this study, PAX8 expression was also significantly downregulated in PTC tissues and PTC cells than that in adjacent healthy tissues and normal cells. In addition, lncRNA PAX8-AS1:28 expression was found to be positively correlated with the expression of PAX8. Moreover, lncRNA PAX8-AS1:28 silencing reduced the expression level of PAX8 and promoted PTC cell growth. In contrast, lncRNA PAX8-AS1:28 overexpression increased the expression level of PAX8 and inhibited PTC cell growth. Those data suggest that PAX8-AS1:28 may affect PTC cell growth by positively regulating the expression of PAX8.

As an oncogene, MYC is overexpressed in various tumor tissues, and MYC overexpression promotes the proliferation, migration and invasion of tumor cells (21). An increased expression level of MYC has also been detected in patients with PTC (22), indicating the involvement of MYC in this disease. Consistent with previous studies, in our study, the expression level of MYC in PTC cells was found to be significantly higher than that in a normal cell line. In a recent study, protein levels of MYC and PAX8 were found to be inversely correlated with each other in thyroid tumors (23), indicating the opposite roles of those 2 proteins in thyroid cancer. In our study, MYC silencing increased expression levels of PAX8-AS1:28 as well as PAX8 and inhibited tumor cell growth, while MYC overexpression decreased expression levels of PAX8-AS1:28 as well as PAX8 and promoted tumor cell growth. MYC is multifunctional and nuclear phosphoprotein that is involved in the expression regulation of a large set of genes. The downregulation of PAX8-AS1:28 and PAX8 may be caused by the direct role of MYC or its downstream targets. All these data suggest that MYC can promote PTC, and this function may be correlated with the downregulation of expression of PAX8-AS1:28 and PAX8. It is interesting that antisense PAX8-AS1:28 elevated the expression of PAX8. The possible explanation is that PAX8-AS1:28 may be involved in the post-transcriptional process of PAX 8 transcripts, which has been observed in a recent study (24).

In conclusion, the expression level of PAX8-AS1:28 and PAX8 were lower in PTC tumor tissue and PTC cell lines than that in healthy tissue and normal cell lines, while the expression level of MYC was higher in PTC cell lines than that in normal cell lines. PAX8-AS1:28 can positively regulate PAX8 expression and inhibit PTC tumor cell growth. In contrast, MYC can negatively regulate the expression of PAX8-AS1:28 as well as PAX8 and promote tumor cell growth. Therefore, PAX8-AS1:28 and PAX8 may serve as biomarkers for the diagnosis of PTC. They may also serve as targets for the treatment of this disease. The present study was still limited by the small sample size. Further studies with a larger sample size are needed to further confirm the conclusions in the present study.

\section{Acknowledgments}

Not applicable.

\section{Funding}

We thank the financial support from Natural Science Foundation of Zhejiang Province (no. LY14H190003).

\section{Availability of data and materials}

The datasets used during the present study are available from the corresponding author upon reasonable request.

\section{Authors' contributions}

YZ and JC conceived and designed the study. YZ and FL performed the experiments. JC wrote the paper. $\mathrm{YZ}$ and $\mathrm{FL}$ reviewed and edited the manuscript. All authors read and approved the manuscript and agree to be accountable for all aspects of the research in ensuring that the accuracy or integrity of any part of the work are appropriately investigated and resolved.

\section{Ethics approval and consent to participate}

The Ethics Committee of the Second Affiliated Hospital of Wenzhou Medical University (Wenzhou, China) approved this study and all patients provided informed consent.

\section{Patient consent for publication}

Not applicable.

\section{Competing interests}

The authors state that they have no competing interests.

\section{References}

1. Agrawal N, Akbani R, Aksoy BA, Ally A, Arachchi H, Asa SL, Auman JT, Balasundaram M, Balu S, Baylin SB, et al: Integrated genomic characterization of papillary thyroid carcinoma. Cell 159: 676-690, 2014.

2. Olaleye O, Ekrikpo U, Moorthy R, Lyne O, Wiseberg J, Black M and Mitchell D: Increasing incidence of differentiated thyroid cancer in South East England: 1987-2006. Eur Arch Otorhinolaryngol 268: 899-906, 2011.

3. Londero SC, Krogdahl A, Bastholt L, Overgaard J, Trolle W, Pedersen HB, Bentzen J, Schytte S, Christiansen P and Godballe C: Papillary thyroid microcarcinoma in Denmark 1996-2008: A national study of epidemiology and clinical significance. Thyroid 23: 1159-1164, 2013.

4. Hay ID, Thompson GB, Grant CS, Bergstralh EJ, Dvorak CE, Gorman CA, Maurer MS, McIver B, Mullan BP, Oberg AL, et al: Papillary thyroid carcinoma managed at the Mayo Clinic during six decades (1940-1999): Temporal trends in initial therapy and long-term outcome in 2444 consecutively treated patients. World J Surg 26: 879-885, 2002

5. Ito Y, Miyauchi A, Kudo T, Kihara M, Fukushima M and Miya A: The effectiveness of prophylactic modified neck dissection for reducing the development of lymph node recurrence of papillary thyroid carcinoma. World J Surg 41: 2283-2289, 2017.

6. Perkel JM. Visiting 'Noncodarnia'. Biotechniques 54: 303-304, 2013.

7. Esteller M: Non-coding RNAs in human disease. Nat Rev Genet 12: 861-874, 2011. 
8. Wang K, Liu F, Zhou LY, Long B, Yuan SM, Wang Y, Liu CY, Sun T, Zhang XJ and Li PF: The long noncoding RNA CHRF regulates cardiac hypertrophy by targeting miR-489. Circ Res 114: 1377-1388, 2014.

9. Takahashi K, Yan I, Haga H and Patel T: Long noncoding RNA in liver diseases. Hepatology 60: 744-753, 2014.

10. Prensner JR and Chinnaiyan AM: The emergence of lncRNAs in cancer biology. Cancer Discov 1: 391-407, 2011.

11. Lan X, Zhang H, Wang Z, Dong W, Sun W, Shao L, Zhang T and Zhang D: Genome-wide analysis of long noncoding RNA expression profile in papillary thyroid carcinoma. Gene 569: $109-117,2015$.

12. Xing M: Molecular pathogenesis and mechanisms of thyroid cancer. Nat Rev Cancer 13: 184-199, 2013.

13. Zerilli M, Zito G, Martorana A, Pitrone M, Cabibi D, Cappello F, Giordano C and Rodolico V: BRAF ${ }^{\mathrm{V} 600 \mathrm{E}}$ mutation influences hypoxia-inducible factor-1 $\alpha$ expression levels in papillary thyroid cancer. Mod Pathol 23: 1052-1060, 2010.

14. Boelaert K, Horacek J, Holder RL, Watkinson JC, Sheppard MC and Franklyn JA: Serum thyrotropin concentration as a novel predictor of malignancy in thyroid nodules investigated by fine-needle aspiration. J Clin Endocrinol Metab 91: 4295-4301, 2006.

15. Clevers $H$ and Nusse $R$ : Wnt/ $\beta$-catenin signaling and disease. Cell 149: 1192-1205, 2012.

16. Xiang C, Zhang ML, Zhao QZ, Xie QP, Yan HC, Yu X, Wang P and Wang Y: LncRNA-SLC6A9-5:2: A potent sensitizer in ${ }^{131}$ I-resistant papillary thyroid carcinoma with PARP-1 induction. Oncotarget 8: 22954-22967, 2017.

17. Wang X, Lu X, Geng Z, Yang $G$ and Shi Y: LncRNA PTCSC3/miR-574-5p governs cell proliferation and migration of papillary thyroid carcinoma via Wnt/ $\beta$-catenin signaling. J Cell Biochem 118: 4745-4752, 2017.
18. Yu V, Singh P, Rahimy E, Zheng H, Kuo SZ, Kim E, Wang-Rodriguez J and Ongkeko WM: RNA-seq analysis identifies key long non-coding RNAs connected to the pathogenesis of alcohol-associated head and neck squamous cell carcinoma. Oncol Lett 12: 2846-2853, 2016.

19. Luo YH, Liang L, He RQ, Wen DY, Deng GF, Yang H, He Y, Ma W, Cai XY, Chen JQ, et al: RNA-sequencing investigation identifies an effective risk score generated by three novel lncRNAs for the survival of papillary thyroid cancer patients. Oncotarget 8: 74139-74158, 2017.

20. Plachov D, Chowdhury K, Walther C, Simon D, Guenet JL and Gruss P: Pax8, a murine paired box gene expressed in the developing excretory system and thyroid gland. Development 110: 643-651, 1990

21. Wolfer A and Ramaswamy S: MYC and metastasis. Cancer Res 71: 2034-2037, 2011.

22. Hu YJ, Luo XY, Yang Y, Chen CY, Zhang ZY and Guo X: Characterization and significance of MUC1 and c-myc expression in elderly patients with papillary thyroid carcinoma. Genet Mol Res 14: 15325-15330, 2015.

23. Zhu X, Zhao L, Park JW, Willingham MC and Cheng SY: Synergistic signaling of KRAS and thyroid hormone receptor $\beta$ mutants promotes undifferentiated thyroid cancer through MYC up-regulation. Neoplasia 16: 757-769, 2014.

24. Van der Wal E, Bergsma AJ, Pijnenburg JM, Van der Ploeg AT and Pijnappel WWMP: Antisense oligonucleotides promote exon inclusion and correct the common c.-32-13T $>$ G GAA splicing variant in pompe disease. Mol Ther Nucleic Acids 7: 90-100, 2017. 